ARTIGO ORIGINAL

\title{
Efeitos da exploração de impacto reduzido (EIR) na composição florística e estrutura de uma floresta ombrófila densa na Amazônia Brasileira
}

\author{
Effects of reduced impact logging (RIL) on the floristic composition and \\ structure of a dense ombrophylous forest in the Brazilian Amazon
}

\author{
Bruno de Almeida Lima ${ }^{1}$ (D), Marcos Felipe Nicoletti ${ }^{1}$ (D), Thiago Floriani Stepka ${ }^{1}$ (D), \\ Samuel de Pádua Chaves e Carvalho² (1), Lia de Oliveira Melo ${ }^{3}$ (1) , Girlene da Silva Cruz ${ }^{1}$ (1) \\ ${ }^{1}$ Universidade do Estado de Santa Catarina - UDESC, Lages, SC, Brasil \\ 2Universidade Federal de Mato Grosso - UFMT, Cuiabá, MT, Brasil \\ ${ }^{3}$ Universidade Federal do Oeste do Pará - UFOPA, Santarém, PA, Brasil
}

Como citar: Lima, B. A., Nicoletti, M. F., Stepka, T. F., Carvalho, S. P. C., Melo, L. O., \& Cruz, G. S. (2021). Efeitos da exploração de impacto reduzido (EIR) na composição florística e estrutura de uma floresta ombrófila densa na Amazônia Brasileira. Scientia Forestalis, 49(131), e3635. https://doi.org/10.18671/scifor.v49n131.23

\begin{abstract}
Resumo
Neste estudo objetivou-se avaliar a mudanças ocorridas composição florística, diversidade de espécies e estrutura da floresta submetida a exploração de impacto reduzido na Floresta Nacional do Tapajós, Pará. Os dados foram obtidos através de parcelas permanentes $\left(2500 \mathrm{~m}^{2}\right)$ instaladas em 3 Unidades de Produção Anual (UPA n² 2, 3 e 5) que foram exploradas em 2007, 2008 e 2010 nas respectivas UPAs. Os inventários ocorreram em 4 ocasiões, sendo a primeira antes e após as explorações. Foi coletado o diâmetro altura do peito medido à 1,30 m do nível solo (dap) igual ou superior a $10 \mathrm{~cm}$. Posteriormente avaliou-se a composição florística, a diversidade por meio do índice de Shannon-Wiener ( $\left.\mathrm{H}^{\prime}\right)$, estimadores fitossociológicos (Densidade, Área basal e Volume). Após, foi realizada a Análise de variância (ANOVA) das variáveis analisadas entre as sucessivas medições a $95 \%$ de probabilidade e em caso de diferença utilizou-se o teste de Tukey $(a=0,05)$. Durante todo o período avaliado foram registrados uma média de 135, 160, 159 espécies nas UPAs 2, 3 e 5 respectivamente. A família com maior riqueza florística foi a Fabaceae em todas as medições nas 3 UPAs. $O$ índice de diversidade $\left(H^{\prime}\right)$ foi considerado alto mesmo após a exploração de impacto reduzido com valores variando de 4,10 a 4,35. Devido a exploração os estimadores avaliados tiveram quedas em seus valores, recuperando-se no decorrer dos anos. A ANOVA indicou diferença significativa apenas na densidade (árvore/ha) na UPA 2. A exploração aplicada nas UPAs minimizou os danos às árvores remanescentes devido a intensidade moderada de corte. $\mathrm{O}$ comportamento da floresta permite inferir que sua recuperação, aos valores pré EIR, seja atingida antes do próximo, dessa forma não comprometendo sua sustentabilidade de produção madeireira.
\end{abstract}

Palavras-chave: Manejo florestal sustentável; Inventário contínuo; Floresta terra firme.

\begin{abstract}
This study aimed to evaluate the changes that occurred in floristic composition, species diversity and forest structure after subjecting the forest to reduced impact exploration in the Tapajos National Forest, Pará. The data were obtained in permanent plots $\left(2500 \mathrm{~m}^{2}\right)$ installed in 3 Annual Production Units (APU $\mathrm{n}^{\circ}$ 2, 3 and 5) that were explored in 2007, 2008 and 2010 in APUs. The Inventories occurred on 4 occasions: the first before logging and the remaining after each logging. Data collected were the diameter at breast height, measured at $1.3 \mathrm{~m}$ above the soil (dbh) equal to or greater than $10 \mathrm{~cm}$. Subsequently the floristic composition and diversity was evaluated using the Shannon-Wiener index ( $\mathrm{H}$ ') by phytosociological estimators (Density, Basal Area and Volume). Analyses of variance (ANOVA) of the
\end{abstract}

Fonte de financiamento: Nenhuma.

Conflito de interesse: Nada a declarar.

Autor correspondente: bruno.lima.stm@hotmail.com

Recebido: 6 novembro 2020.

Aceito: 5 abril 2021

Editor: Paulo Henrique Müller Silva.

(c) (i) Este é um artigo publicado em acesso aberto (Open Access) sob a licença Creative Commons Attribution, que permite uso, distribuição e eprodução em qualquer meio, sem restrições desde que o trabalho original seja corretamente citado. 
analyzed variables were calculated between the successive settings to $95 \%$ of probability; where significant differences were found the Tukey test $(\alpha=0.05)$ was applied. Throughout the evaluated period, an average of 135, 160, 159 species were recorded in APUs 2, 3 and 5, respectively. The family with the greatest floristic wealth was Fabaceae in all registrations in the 3 APUs. The diversity index (H ') was considered high even after the EIR with values ranging from 4.10 to 4.35 . After logging, the estimators obtained dropped in values, recovering over the years. The ANOVA indicated a significant difference only in the tree density (tree.ha-1) in the APUs 2. The logging applied in the APUs minimized the damage to the remaining trees due to the moderate cutting intensity. The behavior of the forest allows us to infer that its recovery to pre-sustainability EIR values, is achieved ahead of the next cutting cycle; therefore not compromising the timber production.

Keywords: Sustainable forest management; Continuous inventory; Upland forest.

\section{INTRODUÇÃO}

A Amazônia possui umas das maiores florestas tropicais do mundo e detêm grande quantidade de recursos naturais (Cardoso et al., 2017; Pereira et al., 2010). É caracterizada pela alta diversidade de espécies com estimativa de existir aproximadamente de 12.000 a 16.000 espécies de árvores em toda a floresta amazônica (Demarchi et al., 2018; Ter Steege et al., 2013).

E para que haja uma exploração seletiva das espécies comerciais devem ser adotadas técnicas que garantam um próximo ciclo de corte e a conservação da floresta remanescente. A alternativa é a exploração de impacto reduzido (EIR)que é uma das ferramentas do manejo florestal, e que pode ser considerado uma técnica para o uso sustentável dos recursos florestais (Amaral et al., 2019). A EIR consiste na implementação de uma série de diretrizes pré e pós-exploração, desenhadas para proteger a regeneração, minimizar os danos ao solo, prevenir danos desnecessários às espécies que não serão exploradas, para manter a estrutura florestal mais semelhante possível às condições pré-extração (Schwartz et al., 2012). No esforço para promover o manejo florestal sustentável a legislação ambiental definiu uma exploração máxima de $30 \mathrm{~m}^{3} \mathrm{ha}^{-1}$ quando mecanizada e um ciclo de corte até 35 anos (Lima et al., 2021).

Toda intervenção planejada em floresta tropical necessita de inventários minuciosos para geração de estimativas precisas dos parâmetros de diversidade, densidade, volume área basal, distribuição diamétrica (Souza et al., 2006). Essas informações serão ferramentas importantes no planejamento e execução do manejo florestal para conduzir essa floresta a uma estrutura balanceada e na escolha de tomadas decisões adequadas a fim de conduzi-las ao bom crescimento (Vieira et al., 2014). O estudo sobre composição florística é importante para conhecer a estrutura da vegetação, que vai possibilitar obter informações qualitativas e quantitativas do local monitorado. E por mais que as técnicas utilizadas na exploração de impacto reduzido danos nas florestas nativas do que exploração convencional predatória, ainda assim os efeitos precisam ser minuciosamente avaliados, e isso requer informações como, citado anteriormente, qualitativa e quantitativa das espécies e da condução das atividades realizadas na exploração. Nesse sentido é imprescindível a análise fitossociológica e monitoramento da floresta para diagnosticar os impactos causados pelas ações no manejo e sua capacidade de recuperação para a próxima exploração (Hirai et al., 2007; Reis et al., 2013).

Apesar de promover menor impacto à vegetação, as técnicas adotadas pelo manejo florestal ainda são uma intervenção na floresta causando mais distúrbios se comparadas aos danos naturais. Pois deve-se considerar que o manejo florestal implica manipular as estruturas e composições das florestas para favorecer algumas espécies, as mudanças são inevitáveis, mas sem causar prejuízos a densidade de árvores, área basal e volume a no médio prazo (Andrade et al., 2020; Avila et al., 2015). Os benefícios da exploração de impacto reduzido podem ser limitados pela intensidade de exploração aplicada e os danos, interferindo também na capacidade de recuperação da floresta (Sist \& Ferreira, 2007). Devido a isso torna-se essencial acompanhar o comportamento da floresta manejada ao longo dos 
anos e avaliar se as técnicas empregadas estão sendo suficientes para a manutenção e recuperação da vegetação remanescente.

Diante desse contexto, o objetivo deste estudo foi avaliar as mudanças ocorridas na composição florística, diversidade e estrutura da floresta em três unidades de produção anual submetidas à exploração de impacto reduzido na Floresta Nacional do Tapajós, Pará.

\section{MATERIAL E MÉTODOS}

\section{Área do estudo}

Este estudo foi realizado no Centro Nacional do Tapajós Floresta (Flona Tapajós), Unidade de Conservação Federal (UC) localizada em oeste do Estado do Pará às margens da rodovia Santarém-Cuiabá (BR-163), localizada nos municípios de Belterra, Aveiro, Placas e Rurópolis, entre as coordenadas geográficas $2^{\circ} 45$ a $4^{\circ} 10^{\prime}$ S e $54^{\circ} 45^{\prime}$ a $55^{\circ} 30^{\prime} \mathrm{W}$ (Santos et al., 2019). A UC ocupa uma área de aproximadamente 544.927 hectares. Na região há predominância de Latossolo Amarelo Distrófico, caracterizados por textura muito argilosa, perfil profundo e baixa fertilidade (Oliveira Junior et al., 2015).

A vegetação é classificada como floresta ombrófila densa, caracterizada pela presença de grandes árvores e pela abundância de cipós lenhosos, palmeiras e epífitas (Instituto Brasileiro de Geografia e Estatística, 2012). A precipitação está concentrada entre janeiro e maio, com precipitação média anual de $1.820 \mathrm{~mm}$, e o clima é classificado como Ami no sistema Köppen (quente e úmido), com temperatura média anual de $25,5^{\circ} \mathrm{C}$ (Alvares et al., 2013).

\section{Coleta e análise de dados}

Os dados foram obtidos a partir das informações do inventário florestal de 26 parcelas permanentes (PP) de 0,25 hectares cada $(50 \mathrm{~m} \times 50 \mathrm{~m}$ ), representando um total de 6,5 ha amostrados, distribuídas de maneira aleatória em três Unidades de Produção Anual (UPA) na área de manejo florestal da Flona Tapajós gerida pela Cooperativa Mista da Flona Tapajós (Coomflona). Todas as árvores com diâmetro altura do peito medido à 1,30 $\mathrm{m}$ do nível do solo (dap) $\geq 10 \mathrm{~cm}$ foram mensuradas conforme as diretrizes de instalação e medição de parcelas permanente proposto por Silva et al. (2005). Em cada UPA foram realizados 4 inventários, sendo um antes da exploração e os três restantes após a exploração. Os inventários das parcelas permanentes da UPA 2 ocorreram nos anos de 2006, 2009, 2012, 2016, enquanto da UPA 3 em 2007, 2010, 2013 e 2016. E por fim a UPA 5 inventariada em 2010, 2011, 2015 e 2016. O total de parcelas e os anos de medições de cada UPA se encontram na Tabela 1.

Tabela 1. Distribuição de parcelas, ano das medições e intensidade de corte em cada Unidade de Produção Anual (UPA), Floresta Nacional do Tapajós.

\begin{tabular}{|c|c|c|c|c|c|}
\hline UPA & $\begin{array}{c}\mathrm{N}^{\circ} \mathrm{de} \\
\text { parcelas }\end{array}$ & Medições & Exploração & $\begin{array}{l}\text { Intensidade de } \\
\text { corte }\left(\mathrm{m}^{3} \mathrm{ha}^{-1}\right)\end{array}$ & $\begin{array}{c}\% \text { do volume } \\
\text { total }\end{array}$ \\
\hline 2 & 6 & $\begin{array}{c}2006 ; 2009 ; 2012 ; \\
2016\end{array}$ & 2007 & 12,17 & 8 \\
\hline 3 & 10 & $\begin{array}{c}2007 ; 2010 ; 2013 ; \\
2016\end{array}$ & 2008 & 15,68 & 9 \\
\hline 5 & 10 & $\begin{array}{c}2010 ; 2011 ; 2015 ; \\
2016\end{array}$ & 2010 & 14,36 & 14 \\
\hline
\end{tabular}

A composição florística foi obtida pela distribuição dos indivíduos em família, gênero e espécies e a diversidade foi calculada mediante o índice de diversidade de Shannon-Wiener $\left(H^{\prime}\right)$ conforme Magurran (1988). A Equabilidade de Pielou (J') foi calculada para representar a uniformidade da distribuição dos indivíduos entre as espécies existentes (Pielou, 1975). 
Os estimadores fitossociológicos da estrutura horizontal (densidade, área basal, índice de valor de importância) usados neste trabalho foram estimados conforme Mueller-Dombois \& Ellenberg (1974). Para a distribuição diamétrica agrupou-se as árvores em classes de diâmetros com amplitude de $10 \mathrm{~cm}$, indicada para floresta tropical amazônica.

Para estimar o volume das árvores foi utilizada a equação desenvolvida para a região dos Tapajós para o inventário contínuo, pela equipe de pesquisadores da Embrapa Amazônia Oriental, a qual usa apenas o dap como variável dependente (Silva et al., 1984; Silva \& Araújo, 1984) (Tabela 2). Em cada UPA todos esses estimadores composição e estrutura florestal serão avaliados nos inventários pré e pós exploração, para serem comparados.

Tabela 2. Equações de volume desenvolvidas para inventário contínuo na Floresta Nacional do Tapajós.

\begin{tabular}{cccc}
\hline Árvores & Equação & $\mathbf{R}^{\mathbf{2}}$ & Syx(\%) \\
\hline $10 \mathrm{~cm} \leq$ dap $<44,9 \mathrm{~cm}$ & $\mathrm{v}=0,0994+9,1941 * 10^{-4} \mathrm{dap}^{2}$ & 0,96 & 12 \\
dap $\geq 45,0 \mathrm{~cm}$ & $\mathrm{Lnv}=-7,6281+2,1809 \ln (\mathrm{dap})$ & 0,84 & 16 \\
\hline
\end{tabular}

$\mathrm{v}=$ volume individual total $\left(\mathrm{m}^{3}\right)$; dap= diâmetro à altura do peito; $\mathrm{R}^{2}=$ coeficiente de determinação; Syx (\%)= erro padrão da estimativa.

No inventário contínuo da UPA 2 foram contabilizadas 4 árvores exploradas, isso representou uma densidade de 3 árv ha-1, um volume $14,1 \mathrm{~m}^{3} \mathrm{ha}^{-1}$, e área basal de 1,1 $\mathrm{m}^{2}$ ha

1. Na UPA 3 a EIR extraiu 9 árvores correspondendo a 4 árv/ha, 2,3 $\mathrm{m}^{2} / \mathrm{ha}$ de área basal, e volume de $32,9 \mathrm{~m}^{3} \mathrm{ha}^{-1}$. O mesmo número de árvores exploradas também foi registrado na UPA 5 que significou 3 árv/ha, 1,7 $\mathrm{m}^{2} \mathrm{ha}^{-1}$ área basal e volume de $24,1 \mathrm{~m}^{3} \mathrm{ha}^{-1}$

\section{Análise estatística}

Aplicou-se para cada UPA a Análise de Variância (ANOVA) à 95\% de probabilidade para avaliar se há diferença estatística entre os valores médios dos estimadores (Área basal, Densidade, Volume, $\mathrm{H}^{\prime}, \mathrm{J}$ ) nas medições. Constatada essa diferença foi aplicada o Teste de médias de Tukey $(\alpha=0,05)$ para verificar quais médias diferem entre si. A normalidade dos dados para atender as premissas da ANOVA foi efetuada através do teste Shapiro-Wilk com 95\% de probabilidade (Razali \& Wah, 2011).

A análise estatística foi realizada no ambiente R (R Development Core Team, 2019) através do pacote de dados ExpDes.pt versão 1.2 (Ferreira et al., 2018). Com o uso do software realizou-se também os seguintes procedimentos: visualização dos dados através dos gráficos de tendência e boxplots que demonstram a dispersão e comportamento dos estimadores como Densidade (árv ha-1), Área basal $\left(\mathrm{m}^{2}\right.$ ha-1 $)$, Volume $\left(\mathrm{m}^{3} \mathrm{ha}^{-1}\right)$, Equabilidade de Pielou (J), Diversidade de Shannon-Wiener $\left(\mathrm{H}^{\prime}\right)$.

\section{RESULTADOS E DISCUSSÃO}

\section{Composição florística}

Durante todo o período avaliado em cada UPA, foram registradas médias de 135, 160, 159 espécies nas UPAs 2, 3 e 5 respectivamente. Na Tabela 3 encontram-se os números detalhados às mudanças na composição florística para cada unidade de produção no respectivo ano de medição.

No levantamento das 26 parcelas considerando árvores com dap acima de $10 \mathrm{~cm}$ foi verificado a grande quantidade de espécies nas UPAs antes da exploração. No segundo inventário, que é a medição seguinte após a exploração, é verificada a queda no número de espécies e gênero em relação ao primeiro inventário (pré-exploração), em todas as unidades de produção (Tabela 3). Isso é consequência das implicações da exploração madeireira que 
provoca danos às árvores remanescentes, podendo levar à letalidade de algumas destas, reduzindo, portanto, o número desses grupos. Mas ao avaliar as medições seguintes notamse a recuperação ingresso de espécies, chegando na última medição com balanço positivo na riqueza florística nas Unidades de Produção, devido a abertura do dossel que estimula a entrada de novas espécies na comunidade. Essas diminuições logo após a exploração e aumento ao longo do tempo foram também observadas em outras pesquisas na Amazônia (Jardim \& Quadros, 2016; Vieira et al., 2014). Os autores relatam que essas variações na composição florística se devem principalmente ao tamanho e a quantidade de aberturas de clareiras no dossel. Já que a abertura de clareiras contribui para formação de novas ecounidades, ou seja, mudanças de espécies nas diferentes categorias sucessionais.

Tabela 3. Composição florística, diversidade de Shannon ( $\left.H^{\prime}\right)$ e equabilidade de Pielou (J) de 3 Unidades de Produção Anual (UPA) em 4 ocasiões, na Floresta Nacional do Tapajós, Pará.

Table 3. Floristic composition, Shannon diversity index $\left(\mathrm{H}^{\prime}\right)$ and Pielou $(\mathrm{J})$ equability of 3 Annual Production Units (APU) on 4 occasions, in the Tapajos National Forest, State of Pará.

\begin{tabular}{|c|c|c|c|c|c|}
\hline \multirow{2}{*}{ UPA } & \multirow{2}{*}{$\begin{array}{c}\text { Grupos } \\
\text { avaliados }\end{array}$} & \multicolumn{4}{|c|}{ Ano das medições } \\
\hline & & 2006 (AE) & 2009 & 2012 & 2016 \\
\hline & Família & 38 & 38 & 38 & 36 \\
\hline \multirow[t]{6}{*}{2} & Gênero & 85 & 83 & 87 & 80 \\
\hline & $\mathrm{S}$ & 131 & 130 & 138 & 140 \\
\hline & $\mathrm{H}^{\prime}$ & $4,1421 a$ & $4,1420 a$ & $4,2192 a$ & $4,2400 a$ \\
\hline & $J$ & $0,8496 a$ & 0,8510a & $0,8563 a$ & $0,8574 a$ \\
\hline & & 2007 (AE) & 2010 & 2013 & 2016 \\
\hline & Família & 45 & 45 & 44 & 43 \\
\hline \multirow[t]{6}{*}{3} & Gênero & 107 & 105 & 106 & 100 \\
\hline & $\mathrm{S}$ & 159 & 155 & 163 & 164 \\
\hline & $\mathrm{H}^{\prime}$ & $4,3302 a$ & 4,3033a & $4,3771 a$ & $4,3904 a$ \\
\hline & $J$ & $0,8543 a$ & $0,8532 a$ & $0,8593 a$ & $0,8609 a$ \\
\hline & & 2010 (AE) & 2011 & 2015 & 2016 \\
\hline & Família & 39 & 39 & 39 & 39 \\
\hline \multirow[t]{4}{*}{5} & Gênero & 100 & 100 & 101 & 98 \\
\hline & $\mathrm{S}$ & 160 & 158 & 159 & 160 \\
\hline & $\mathrm{H}^{\prime}$ & 4,3451a & $4,3438 a$ & $4,3560 a$ & $4,3573 b$ \\
\hline & $J$ & $0,8561 a$ & $0,8580 a$ & 0,8593a & $0,8596 a$ \\
\hline
\end{tabular}

S: riqueza de espécies; H': Shannon-Wiener; J: Equabilidade de Pielou. AE: inventário Antes da Exploração. Letras iguais na horizontal não diferem estatisticamente pelo teste Tukey $(a=0,05)$.

A UPA 2 teve o maior aumento com acréscimo de 13 espécies, seguido da UPA 3 e a UPA 5 que manteve estável sua riqueza.

A composição florística mostra que as áreas avaliadas possuem elevado número espécies, tanto antes e após o manejo aplicado em conformidade com outros trabalhos na região por exemplo Almeida et al. (2012) e Condé \& Tonini (2013). De modo geral as intervenções da exploração causaram distúrbios modificando a composição florística e estrutura da floresta. Dessa forma pode-se inferir que o manejo florestal resultou ao longo dos anos após a exploração no aumento do número de espécies, onde na medição final em cada UPA a riqueza florística teve um balanço positivo, com maior quantidade de espécies que a medição inicial.

As famílias com as maiores riquezas nas UPAs foram Fabaceae, Lauraceae, Sapotaceae, Lecythidaceae, Moraceae, Burseraceae, Euphorbiaceae, Myrtaceae e Flaucourtiaceae tanto antes como após a exploração. Essas famílias concentraram maior número de espécies e indivíduos, juntas representaram em torno de $60 \%$ do total inventariado. Esse 
comportamento de poucas famílias concentrarem grande parte das espécies é um padrão característico da região amazônica, que também foi verificado por Azevedo (2006), Costa et al. (2002), Lima et al. (2018) que avaliaram a composição florística na Amazônia Oriental.

Analisando as mudanças ocorridas na UPA 2 no período (2006-2009) houve a perda de três e ingresso de duas espécies. As ingressantes nesses anos foram pioneiras (Swartizia polyphylla DC. e Inga capitata Desv.), já que a abertura de clareiras em decorrência da colheita pode favorecer o aparecimento de árvores deste grupo ecológico. Entre os anos 2009 a 2012 na comunidade houve a perda de 2 espécies e o ingresso de 10 espécies e no último período (2012-2016) houve ingresso de 2 espécies.

Na UPA 3 o período entre 2007 e 2010 houve a perda de 7 espécies da medição para outra e no ano de 2010 ocorreu ingresso de 3 espécies e novamente estas que surgiram após o corte seletivo de árvores são dos grupos das pioneiras. Este fato já era esperado por causa do favorecimento de um ambiente favorável a este grupo ecológico. Já no período 2010-2013 ocorreu a perda de uma espécie enquanto ingressaram nove. Na medição de 2013 a 2016 ocorreu apenas ingresso de uma espécie.

Na UPA 5 entre 2010 e 2011 ocorreu a perda de duas espécies e não houve ingresso. Já no período de 2011 a 2015 três espécies que ocorreram na $2^{\circ}$ medição não apareceram na $3^{\circ}$ medição, e nesse período houve o ingresso de 4 novas espécies. No último período (20152016) manteve-se estável o balanço da riqueza sem ingresso a perda de espécies.

De maneira geral o que se pode observar é que a intervenção ocasionou mudanças na composição florística decorridas da exploração, aumentando a riqueza de espécies pioneiras, esse mesmo comportamento é visto por Silva et al. (1995) que verificou na região do Tapajós (Pará) que a extração seletiva de madeira alterou a dinâmica florística que reduziu o número de espécies tolerantes (grupo ecológico que apresenta crescimento lento, tolerantes ao sombreamento e atingem o dossel da floresta) em relação ao período anterior da EIR e estimulou o aparecimento das pioneiras logo após a exploração. A redução do número de espécies após a extração seletiva de árvores é verificada por Lima et al. (2018) que ao avaliarem o efeito do manejo florestal na estrutura da vegetação observou a redução da riqueza florística em comparação com a floresta sem intervenção. Silva (2004) no município de Paragominas-PA, registrou no primeiro inventário um total de 157 espécies e após a EIR esse número teve redução de 6\%, e 7 anos após o corte este número ainda se mantinha 2,5\% menor em relação a riqueza florística inicial.

O índice de Shannon $\left(\mathrm{H}^{\prime}\right)$ durante o período avaliado nas UPAs teve pouca variação ficando entre 4,14 e 4,40. Na primeira medição (2010) a UPA 5 apresentou maior valor $\mathrm{H}^{\prime}$ com 4,34 e juntamente com a UPA 3 tiveram comportamento similar ao logo dos anos. Como verificado a UPA 2 teve os menores valor de $\mathrm{H}^{\prime}$ em todas as medições em comparação com as demais. É possível observar uma leve atenuação da diversidade de Shannon na medição seguinte após a exploração madeireira em todas as UPAs e em seguida há o aumento desse índice ao longo dos anos, recuperando e ultrapassando os valores registrados incialmente. Bezerra et al. (2018) avaliando a diversidade de uma floresta antes e pós manejo na Flona Tapajós também verificou alto valor de $\mathrm{H}^{\prime}$ na primeira medição (2012) e a diminuição na segunda medição (2015) com valores de 4,8 e 4,5 respectivamente e essas diferenças não foram significativas.

Observa-se que antes e após a intervenção feita na floresta, o índice de Shannon foi elevado nas três unidades de produção monitoradas. As áreas apresentaram altos valores de $H^{\prime}$ acima de 3,5, que segundo Floriano (2014) e Knight (1975) são considerados diversidade elevada para qualquer tipo de vegetação. Conforme verificado houve pequena diminuição no número de espécies da medição 2006 para 2009, entretanto essa mudança não foi suficiente para afetar a diversidade que continuou elevada. Este cenário foi observado por Jacobsen et al. (2020).

O índice equabilidade de Pielou (J) médio entre as medições de cada UPA ficou em torno de 0,85 . O valor de equabilidade teve uma queda na UPA 3 na segunda medição (2010), mas se recuperou 3 anos mais tarde (2013), nas outras unidades de produção continuou a crescer nos inventários posteriores, mesmo após o manejo na área. O comportamento da 
equabilidade da UPA 2 assim como verificado no índice de Shannon e número de espécies, teve os menores valores em relação as outras unidades de produção. O que pode explicar o baixo valor dessas variáveis na UPA 2 em relação as demais foi o número de parcelas inventariadas. Enquanto nessa foram 6 unidades amostrais, nas outras duas UPAs foram 10 parcelas cada. Ribeiro et al. (2013) ao avaliarem a estrutura de uma floresta manejada e não manejada no município de Placas (Pará) obtiveram respectivamente índices de equabilidade Pielou (J) de 0,85 e 0,86, resultados condizentes com os valores deste trabalho.

Pela Análise de Variância não foi detectada diferença significativa no índice de Shannon para UPA 2 (F: 0,81; p-valor=0,5037) e UPA 3 (F: 1,95; p-valor=0,1392). Esse mesmo comportamento foi observado para equabilidade de Pielou onde não ocorreu diferença significativa nos intervalos de tempo em cada UPA, todos com $p$-valor $<0,05$, mostrando que a exploração não contribui para alteração desses parâmetros. A exceção foi a UPA 5 que apresentou diferença significativa entre as medições em relação à diversidade de Shannon (F: 7,73; p-valor=0,0004). Esse resultado ocorre devido a UPA 5, com 6 anos de medições, ainda esteja sofrendo os efeitos da extração seletiva, além do mais está ocorrendo o estabelecimento de novas árvores e essa fase de sucessão está afetando a diversidade.

\section{Estrutura da floresta}

A densidade total da UPA 2 antes da exploração (2006) correspondeu a 453 árv ha-1 ${ }^{-1}$ na $2^{\circ}$ medição (2009) uma redução para 423 árv ha $^{-1}$ e se recuperando nos anos posteriores chegando no ano de 2016 com 470 árv ha $^{-1}$, ou seja, um aumento de 3,7\% nesse intervalo de 10 anos. A área basal variou de 30,4 $\mathrm{m}^{2}$ ha-1 em 2006 até $30,7 \mathrm{~m}^{2}$ ha-1 na medição de 2016 . 0 comportamento do volume foi de $327,1 \mathrm{~m}^{3} \mathrm{ha}^{-1}$ a $329,5 \mathrm{~m}^{3} \mathrm{ha}^{-1}$ representando a primeira e última medição respectivamente, isso é equivalente a um aumento de aproximadamente $1 \%$.

Essas variáveis se recuperaram num período de 10 anos, mostrando boa resposta da floresta à exploração. Embora as variáveis de área basal, volume e densidade diminuam em virtude da exploração e danos, paralelamente essa atividade de manejo contribui para a abertura do dossel o que favorece o ingresso de novas espécies e de árvores de espécies já presentes da área, impulsionando o desenvolvimento das remanescentes, aumentando consequentemente o número de árvores ao longo dos anos.

As experiências mostram que após certo período os crescimentos dessas variáveis se estabilizam à medida que se fecha o dossel, diminuindo a disponibilidade de luz que desacelera o aparecimento de novas árvores (Dionisio et al., 2018; Oliveira et al., 2005). Andrade et al. (2020), monitorando a recuperação de floresta manejada na Flona Tapajós registraram que esse período de estabilização durou 15 anos.

Na UPA 3 a densidade na medição inicial (2007) correspondeu um total de 463 árvores/ha e na final (2016) de 472 árv ha-1 (+2\%), a área basal passou de $31,5 \mathrm{~m}^{2} \mathrm{ha}^{-1}$ a $29,9 \mathrm{~m}^{2} \mathrm{ha}^{-1}$, uma queda de $7,62 \%$. Em termos de volume antes da exploração contabilizou-se um total de 334,8 $\mathrm{m}^{3} \mathrm{ha}^{-1}$ para $300,5 \mathrm{~m}^{3} \mathrm{ha}^{-1}$, ou seja, diminuição de $10,25 \%$.

Na UPA 5 comparando a primeira e última medição (2010-2016), houve um aumento de $9 \%$ na densidade, a área basal aumentou $6 \%$, e no volume ocorreu uma queda de $5 \%$ (Tabela 4).

O fato da UPA 5 ter seis anos de monitoramento (2010-2016) contribuiu para que o volume do último ano ainda esteja abaixo da medição 2010. Este estudo tem 4 medições para cada UPA e a área mais antiga tem 10 anos de monitoramento.

O comportamento das variáveis densidade, área basal e volume foi parecido com outras práticas de manejo correlatas na Flona Tapajós, ou seja, recuperando seu estoque e mesmo para aquelas que ainda não recuperaram, mas caminham em direção às condições originais, pois após a EIR essas variáveis estão apresentando crescimento considerável. Estes fatos corroboram a vantagem da aplicação da exploração de impacto reduzido na Amazônia, no caso desse trabalho as áreas de monitoramento que foram submetidas a intensidade permitida pela legislação conseguiram manter resiliência e mostrando-se capazes de se restabelecerem tendo transcorrido no máximo 10 anos para um ciclo de corte de 25-30 anos. 
Tabela 4. Número de árvores total por hectare, Área Basal (G) e Volume (V) em cada UPA em 4 períodos, na Floresta Nacional do Tapajós, Pará.

\begin{tabular}{cccccc}
\hline UPA & medição & $\mathbf{N} / \mathbf{h a}$ & $\mathbf{G}\left(\mathbf{m}^{\mathbf{2}} \mathbf{h} \mathbf{a}^{-\mathbf{1}} \mathbf{)}\right.$ & $\mathbf{V}\left(\mathbf{m}^{\mathbf{3}} \mathbf{h} \mathbf{a}^{-\mathbf{1}}\right)$ & $\begin{array}{c}\mathbf{N}^{\circ} \text { total de } \\
\text { árvores }\end{array}$ \\
\hline \multirow{2}{*}{2} & 2006 & 453 & 30,4 & 327,1 & 680 \\
& 2009 & 423 & 29,4 & 316,7 & 635 \\
& 2012 & 451 & 29,1 & 310,5 & 677 \\
& 2016 & 470 & 30,7 & 329,5 & 705 \\
3 & 2007 & 463 & 31,5 & 334,8 & 1158 \\
& 2010 & 450 & 26,9 & 275,3 & 1127 \\
& 2013 & 459 & 27,0 & 274,6 & 1150 \\
& 2016 & 472 & 29,1 & 300,4 & 1181 \\
5 & 2010 & 399 & 24,1 & 244,9 & 1097 \\
& 2011 & 388 & 22,2 & 220,4 & 1068 \\
& 2015 & 394 & 22,9 & 228,8 & 1083 \\
& 2016 & 436 & 25,6 & 231,9 & 1091 \\
\hline
\end{tabular}

Todos as variáveis reduziram seus valores após a primeira medição em todas as UPAs. Isso foi reflexo da exploração de madeira que consequentemente reduziu esses valores, por causa dos danos dessa atividade às árvores remanescentes. Oliveira et al. (2019) também verificou a mesma tendência em uma floresta ombrófila densa que foi manejada no município de Paragominas-PA Os autores detectaram que após a diminuição de área basal e da densidade a floresta com o decorrer dos anos foi recuperando sua estrutura, chegando 10 anos após a medição com aproximadamente $80 \%$ dos valores da floresta original. Outros autores como Vieira et al. (2014) avaliando as implicações do manejo na Amazônia observaram alterações deste tipo.

A exploração de árvores de grande porte afeta diretamente na diminuição da área basal de uma floresta (Lima et al., 2018). Isso é motivado pelo diâmetro mínimo de corte (DMC) na Amazônia que é de $50 \mathrm{~cm}$, conforme estabelecido pela Instrução Normativa IN n05/2006 MMA (Brasil, 2006), portanto quanto maior o diâmetro de uma árvore maior será a redução da área basal, além do impacto da queda que causará na vegetação remanescente. O que também foi verificado neste trabalho como por exemplo na UPA 3 onde a redução foi de 15\% e seguida da UPA 5 e $2 \mathrm{com} 7 \%$ e $4 \%$ respectivamente. Progressivamente a área basal foi se recuperando, chegando na última medição com valor bem próximo ao encontrado na medição inicial em todas as áreas.

Apesar de não ser o foco do trabalho, mas comparando com a exploração convencional, Vidal et al. (2002) registraram uma redução média em área basal, após a exploração, de aproximadamente $31 \%$ que pode ser considerada uma perda considerável para resiliência da floresta. Os baixos percentuais de redução de área basal do presente trabalho que variaram de $4 \%$ a $15 \%$ são resultantes das técnicas da EIR e estão dentro do esperado para este cenário. Essas técnicas incluíram o planejamento da extração, que compreenderam o inventário e mapeamento das árvores e o treinamento das equipes de colheita.

A tendência do volume seguiu o mesmo comportamento da densidade e área basal apenas na UPA 2, chegando no último ano de inventário com valores maiores comparado ao primeiro inventário. Com exceção da UPA 3 e 5 que no ano de 2016 com os volumes de $300,5 \mathrm{~m}^{3}$ ha $^{-1}$ e $231,9 \mathrm{~m}^{3} \mathrm{ha}^{-1}$ não superaram os valores registrados na primeira medição.

Nos boxplots (Figuras 1, 2, 3) nota-se nitidamente o comportamento dos estimadores em diferentes anos. A variação após a exploração seletiva diminui e em seguida se recupera em todas as variáveis. Para a UPA 2 o número de indivíduos por hectare variou significativamente nos intervalos de tempo pela ANOVA (F: 3,16; $p$-valor $=0,0471)$ e o teste Tukey $(\alpha=0,05)$ evidenciou que o valores após o manejo (medição 2009) não diferiram dos anos de 2006 e 2012, apenas do ano 2016. Para as variáveis área basal (F: 0,52; $\mathrm{p}$-valor $=0,6728$ ) e volume (F: 0,72 ; $p$-valor $=0,5516$ ) não houve diferença significativa (Figura 1). Avaliando cada estimador da UPA 3 todos apresentaram igualdade estatística pela ANOVA e teste Tukey (Figura 2). 

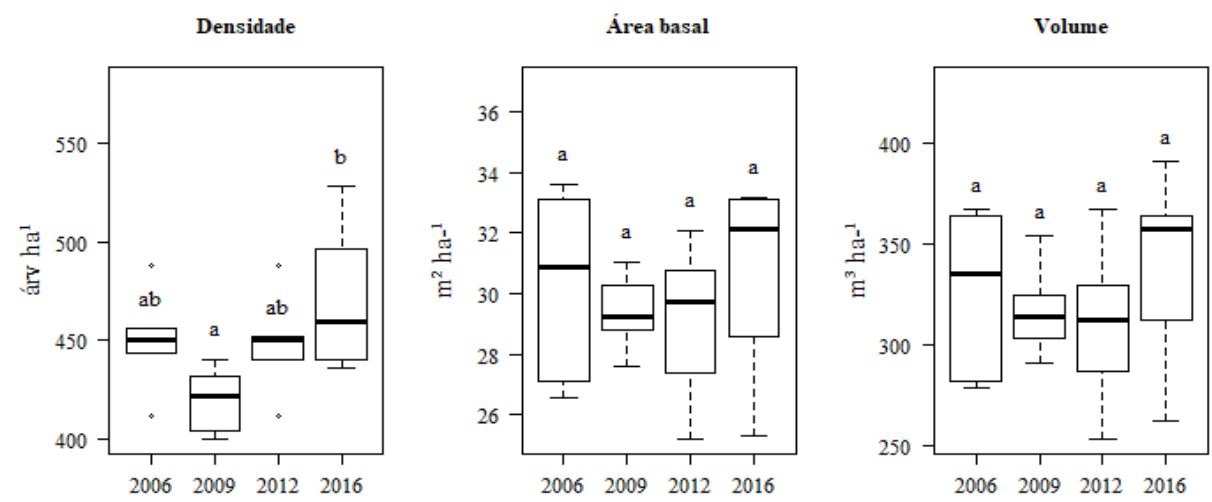

Figura 1 - Boxplot dos estimadores (Densidade, Área basal e Volume) da Unidade de Produção Anual 2, na Floresta Nacional do Tapajós, Pará. Variação de 50\% nos dados entre o primeiro e o terceiro quartis são definidos pela caixa que mostra a mediana (linha horizontal interna), os valores máximo e mínimo (linhas tracejadas). Letras iguais não diferem estatisticamente pelo teste Tukey $(a=0,05)$.
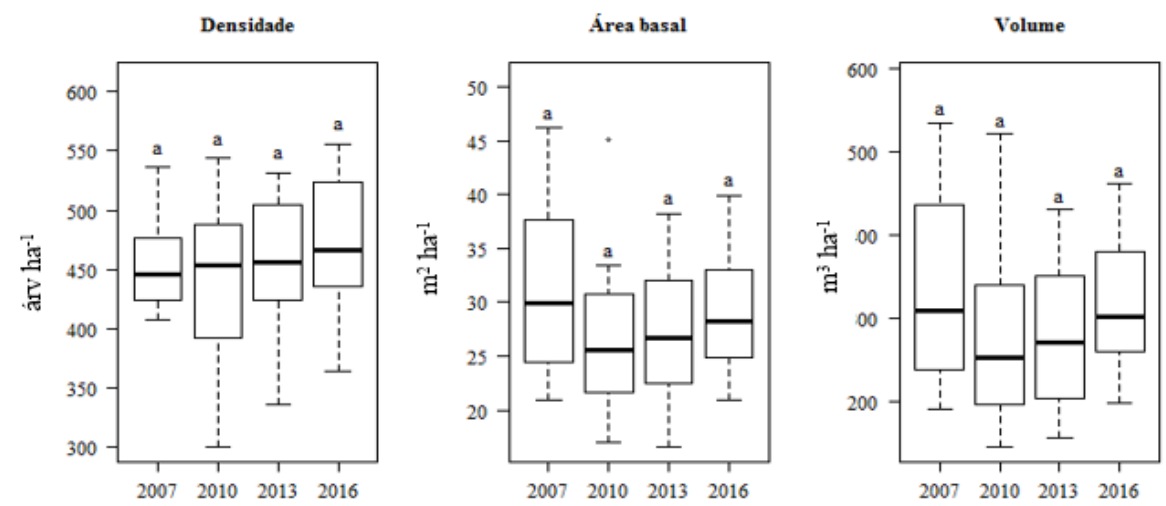

Figura 2. Boxplot dos estimadores (Densidade, Área basal e Volume) da Unidade de Produção Anual Nº 3, na Floresta Nacional do Tapajós, Pará. Variação de $50 \%$ nos dados entre o primeiro e o terceiro quartis são definidos pela caixa que mostra a mediana (linha horizontal interna), os valores máximo e mínimo (linhas tracejadas). Letras iguais não diferem estatisticamente pelo teste Tukey $(\alpha=0,05)$.

A UPA 5 teve diferença estatística apenas na densidade (F: 10,85; $p$-valor $<0,05)$ e o teste Tukey $(a=0,05)$ indicou diferenças significativas entre o valor de 2016 em relação aos anos anteriores (Figura 3).
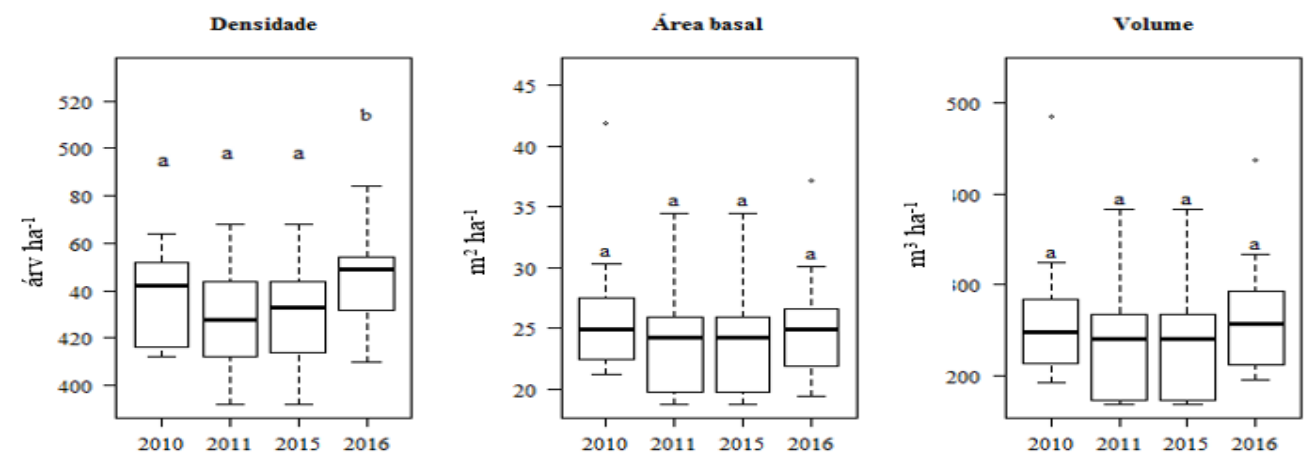

Figura 3. Boxplot dos estimadores (Densidade, Área basal e Volume) da Unidade de Produção Anual 5, na Floresta Nacional do Tapajós, Pará. Variação de 50\% nos dados entre o primeiro e o terceiro quartis são definidos pela caixa que mostra a mediana (linha horizontal interna), os valores máximo e mínimo (linhas tracejadas). Letras iguais não diferem estatisticamente pelo teste Tukey $(a=0,05)$. 
As Figuras 1, 2, 3 mostram que nas UPAs ocorreram flutuações das variáveis ao longo dos anos, mas que em sua maioria não foram significativas, mostrando que a técnica de manejo utilizada é viável para conservação, os danos a vegetação remanescentes são naturalmente suprimidos e a floresta apresenta bons indicadores de recuperação. Nos casos em que houve diferenças significativas, aqui se referindo densidade da UPA 2 e 5, foram motivadas pelas últimas medições nas quais apresentaram valores elevados em relação aos demais anos. Esses resultados deixam claro que a adoção da técnica de EIR pelo manejo florestal provocam sim distúrbios na floresta assim como outras intervenções antrópicas (desmatamento ilegal, queimada, pecuária), com a diferença que o MFS não causa prejuízos tão danosos à estrutura da floresta (densidade área basal e volume) no médio prazo com a retirada de madeira. Isso reforça que as florestas manejadas com técnicas de EIR são recursos capazes de ao mesmo tempo explorarem os recursos disponíveis e garantirem a viabilidade econômica e sustentável.

As 3 UPAS sofreram uma intensidade de exploração moderada, praticamente metade que a Instrução Normativa IN n05/2006 MMA permite (30 $\left.\mathrm{m}^{3} \mathrm{ha}^{-1}\right)$, e isso aliado ao uso das técnicas de EIR como a abertura de estradas planejadas, corte direcionado, pátio de estoque, ajudam a explicar recuperação e o menor efeito nas variáveis já supracitadas. Coincidentemente as áreas que sofreram as maiores intensidades de corte como a UPA 3 com 15,68 $\mathrm{m}^{3}$ ha-1 correspondendo a $9 \%$ do volume total e UPA $5 \mathrm{com} 14,36 \mathrm{~m}^{3} / \mathrm{ha}$ e $14 \%$ do volume total, não recuperaram seu estoque de volume após quase 10 anos da exploração, diferente da UPA 2 (12,17 $\mathrm{m}^{3}$ ha-1 ou $8 \%$ do volume total) que restabeleceu seu volume comparada a medição antes da EIR. Segundo Oliveira et al. (2005) as alterações na floresta estão diretamente ligadas a intensidade das intervenções e com os métodos utilizados. As intensidades moderadas das UPAs permitem inferir que a próxima exploração será antes do ciclo de corte máximo estabelecido pela legislação (35 anos), já que quanto maior a intensidade a ser adotada, maior deverá ser o ciclo de corte, para propiciar tempo suficiente de recuperação a floresta até a próxima exploração (Espada et al., 2015).

Quanto a estrutura diamétrica observou-se uma elevada concentração de árvores nas menores classes de diâmetro e uma diminuição exponencial no número de indivíduos em classes maiores. Isso é a característica de um padrão J-invertido, comportamento que se manteve mesmo após a exploração (Figura 4). Esse padrão é típico de floresta tropical primária (Xaud et al., 2013). Isso sugere que as populações componentes de uma comunidade estão estáveis e autorregenerando devido ao balanço entre mortalidade e recrutamento de árvores (Gonçalves \& Santos, 2008; Phillips et al., 1994).

Ainda na Figura 4 percebe-se que nas menores classes de dap é mais nítida a diminuição e o aumento da densidade antes e após a exploração, e segundo os autores Vatraz et al. (2016) e Rutishauser et al. (2016) dois fatores podem estar relacionados a isso, a saber: $\left(1^{\circ}\right)$ primeiro é que as árvores com os menores dap, mesmo não sendo alvos da extração de madeira, são as mais propensas aos danos da exploração, explicando a diminuição logo após a intervenção. $\left(2^{\circ}\right)$ O segundo motivo é que após a retirada da árvore abre-se uma clareira pequena na floresta. E isso contribuiu para aceleração de seu crescimento e ingresso de novas árvores que atingiram o dap de inclusão $(10 \mathrm{~cm})$, porque essa abertura facilita a entrada de raio solar e favorece as espécies pioneiras.

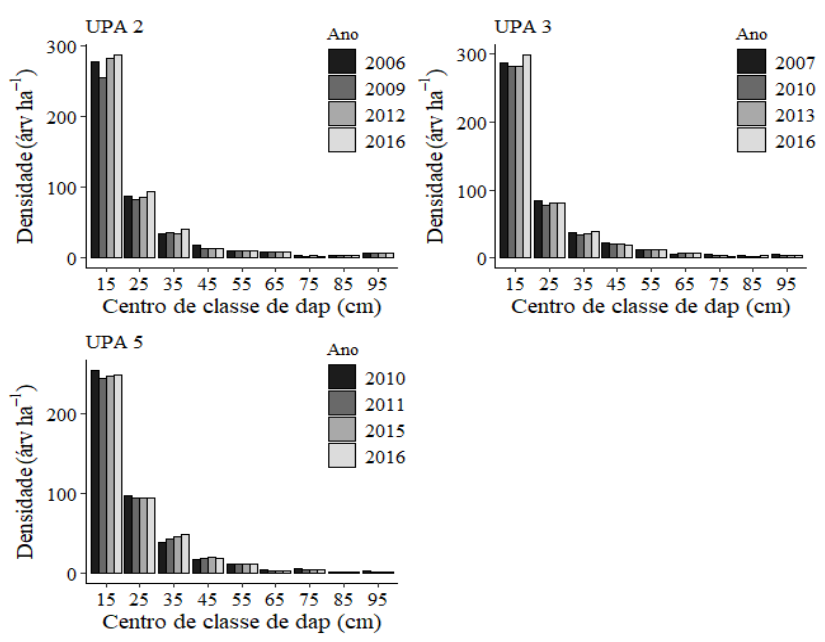

Figura 4. Distribuição diamétrica das árvores mensuradas (árv/ha) em cada medição em 3 diferentes UPAs, na Floresta Nacional do Tapajós, Pará. 
Ao longo dos anos os valores de densidade se recuperaram, como mostrado na Tabela 4, e isso se reflete na estrutura diamétrica. No ano de 2016, que corresponde a 10 e 9 anos após a primeira medição para as UPAS 2 e 3 respectivamente, percebe-se nas classes de diâmetro iniciais a recuperação de densidade, inclusive superior aos valores antes da exploração. Na UPA 5 as classes 15 e $25 \mathrm{~cm}$ no ano de 2016 ainda não recuperaram suas densidades comparadas a 2010, que pode ser justificada pela quantidade de anos transcorrido, que somam 6 anos de medição. Já nas árvores inclusas em algumas classes de dap $>45 \mathrm{~cm}$ no ano de 2016 a densidade está ligeiramente abaixo de períodos anteriores, porque nessas classes houve a exploração de espécies. Ruschel (2008) em área de EIR avaliou a mesma tendência, ou seja, classes de dap $>45$ ainda não tinham recuperado totalmente a densidade comparada às arvores de menor dap, mas que o estoque para o próximo ciclo estava garantido.

Na medição de 2016 em todas a UPAs as maiores quantidades de árvores, em torno de $70 \%$, concentraram-se principalmente nas classes iniciais. Andrade et al. (2015) indica que este fato, mesmo após o manejo florestal, mostra que essa capacidade de regeneração característica de floresta tropical amazônica, ajuda a manter sustentabilidade e a viabilidade da produção florestal. Pois são essas árvores de classes diamétricas inferiores que mudarão para classes superiores ao longo dos anos até que atinja o Diâmetro Mínimo de Corte (DMC) de $50 \mathrm{~cm}$ para exploração.

\section{CONCLUSÃO}

As intervenções realizadas provocaram reduções na riqueza de espécies e na diversidade de Shannon-Wiener e Pielou na primeira medição pós-exploração, mas essas reduções não foram significativas e se recuperaram até o final da medição em cada UPA.

A tendência observada foi a redução não significativa de densidade, área basal e volume (densidade, área basal e volume) avaliados após a EIR e em seguida a recuperação dos valores em relação as medições iniciais, chegando até ultrapassá-los na maioria dos casos.

O manejo aplicado manteve a distribuição diamétrica padrão de florestas nativas em todas as medições, isso mostra que a EIR causou poucos danos nas árvores das classes iniciais.

A exploração aplicada nas três UPAs pôde ser considerada de baixo impacto, minimizou os danos às árvores remanescentes.

\section{REFERÊNCIAS BIBLIOGRÁFICAS}

Almeida, L. S., Gama, J. R. V., Oliveira, F. A., Carvalho, J. O. P., Gonçalves, D. C. M., \& Araújo, G. C. (2012). Fitossociologia e uso múltiplo de espécies arbóreas em floresta manejada, Comunidade Santo Antônio, município de Santarém, Estado do Pará. Acta Amazonica, 42(2), 185-194.

Alvares, C. A., Stape, J. L., Sentelhas, P. C., Gonçalves, J. L. M., \& Sparovek, G. (2013). Koppen's climate classification map for Brazil. Meteorologische Zeitschrift, 22(6), 711-728.

Amaral, M. R., Lima, A. J., Higuchi, F. G., Santos, J., \& Higuchi, N. (2019). Dynamics of tropical forest twenty-five years after experimental logging in Central Amazon mature forest. Forests, $10(2), 89$.

Andrade, D. F. C., Ruschel, A. R., Schwartz, G., Carvalho, J. O. P., Humphries, S., \& Gama, J. R. V. (2020). Forest resilience to fire in eastern Amazon depends on the intensity of pre-fire disturbance. Forest Ecology and Management, 472, 118258.

Andrade, D. F., Gama, J. R. V., Melo, L. O., \& Ruschel, A. R. (2015). Inventário florestal de grandes áreas na Floresta Nacional do Tapajós, Pará, Amazônia, Brasil. Biota Amazônia, 5(1), 109-115.

Avila, A. L., Ruschel, A. R., Carvalho, J. O. P., Mazzei, L., Silva, J. N. M., Lopes, J. C., Araujo, M. M., Dormann, C. F., \& Bauhus, J. (2015). Medium-term dynamics of tree species composition in response to silvicultural intervention intensities in a tropical rain forest. Biological Conservation, 191, 577-586.

Azevedo, C. P. (2006). Dinâmica de florestas submetidas a manejo na Amazônia Oriental: experimentação e simulação (Tese de doutorado). Universidade Federal do Paraná, Curitiba. 
Bezerra, T. G., Lima, A. O. S., Araújo, J. T. R., Santos, M. G. S., Neves, R. L. P., Moraes, G. C., \& Melo, L. O. (2018). Estrutura e dinâmica de uma área manejada na Floresta Nacional do Tapajós. Revista Agroecossistemas, 10(2), 94-112.

Brasil. Ministério do Meio Ambiente. (2006, 5 de março). Instrução normativa n 5, de 11 de dezembro de 2006. Dispõe sobre procedimentos técnicos para elaboração, apresentação, execução e avaliação técnica de Planos de Manejo Florestal Sustentável-PMFSs nas florestas primitivas e suas formas de sucessão na Amazônia Legal, e dá outras providências. Diário Oficial [da] República Federativa do Brasil, Brasília.

Cardoso, D., Särkinen, T., Alexander, S., Amorim, A. M., Bittrich, V., Celis, M., Daly, D. C., Fiaschi, P., Funk, V. A., Giacomin, L. L., Goldenberg, R., Heiden, G., Iganci, J., Kelloff, C. L., Knapp, S., Cavalcante de Lima, H., Machado, A. F. P., Santos, R. M., Mello-Silva, R., Michelangeli, F. A., Mitchell, J., Moonlight P., Moraes, P. L. R., Mori, S. A., Nunes, T. S., Pennington, T. D., Pirani, J. R., Prance, G. T., Queiroz, L. P., Rapini, A., Riina, R., Rincon, C. A. V., Roque, N., Shimizu, G., Sobral, M., Stehmann, J. R., Stevens, W. D., Taylor, C. M., Trovó, M., van den Berg, C., van der Werff, H., Viana, P. L., Zartman, C. E., \& Forzza, R. C. (2017). Amazon plant diversity revealed by a taxonomically verified species list. Proceedings of the National Academy of Sciences of the United States of America, 114(40), 10695-10700.

Condé, T. M., \& Tonini, H. (2013). Fitossociologia de uma Floresta Ombrófila Densa na Amazônia Setentrional, Roraima, Brasil. Acta Amazonica, 43(3), 247-259.

Costa, D. H. M., Carvalho, J. O. P., \& Silva, J. N. M. (2002). Dinâmica da composição florística após a colheita de madeira em uma área de terra firme na Floresta Nacional do Tapajós (PA). Revista de Ciências Agrárias, 38, 67-90.

Demarchi, L. O., Scudeller, V. V., Moura, L. C., Dias-Terceiro, R. G., Lopes, A., Wittmann, F. K., \& Piedade, M. T. F. (2018). Floristic composition, structure and soil-vegetation relations in three white-sand soil patches in central Amazonia. Acta Amazonica, 48(1), 46-56.

Dionisio, L. F. S., Schwartz, G., Lopes, J. C., \& Oliveira, F. A. (2018). Growth, mortality, and recruitment of tree species in an Amazonian rainforest over 13 years of reduced impact logging. Forest Ecology and Management, 430, 150-156.

Espada, A., Pires, T., Lentini, M., \& Bittencourt, P. (2015). Manejo florestal e exploração de impacto reduzido em florestas naturais de produção da Amazônia. Informativo Técnico I IFT. Belém, IFT, p.32.

Ferreira, E. B., Cavalcanti, P. P., \& Nogueira, D. A. (2018). ExpDes.pt: pacote experimental designs (Portuguese). R package version 1.2.0. Recuperado em 6 de novembro de 2020, de https://CRAN.Rproject.org/package=ExpDes.pt

Floriano, E. P. (2014). Fitossociologia florestal (1. ed., 136 p.). São Gabriel: Unipampa.

Gonçalves, F. G., \& Santos, J. R. (2008). Composição florística e estrutura de uma unidade de manejo florestal sustentável na Floresta Nacional do Tapajós, Pará. Acta Amazonica, 38(2), 229-244.

Hirai, E. H., de Carvalho, J. O. P., \& Pinheiro, K. A. O. (2007). Comportamento populacional de cupiúba (Goupia glabra Aublet) em floresta de terra firme na fazenda Rio Capim, Paragominas, PA. Revista de Ciências Agrárias, 47, 89-102.

Instituto Brasileiro de Geografia e Estatística - IBGE. (2012). Manual técnico da vegetação brasileira. (2. ed. ver. amp.). Rio de Janeiro.

Jacobsen, R. H. F., Sccoti, M. S. V., Fagundes, S. T. S., Brito Junior, J. F., \& Biazatti, S. C. (2020). Impacts on vegetation after selective cutting in forest concession area in the southwestern brazilian amazon. Floresta, 50(4), 1778-1787.

Jardim, F. C. S., \& Quadros, L. C. L. (2016). Estrutura de uma floresta tropical dez anos após exploração de madeira em Moju, Pará. Revista Ceres, 63(4), 427-435.

Knight, D. H. (1975). A phytosociological analysis of species-rich tropical forest on Barro Colorado Island, Panamá. Ecological Monographs, 45(3), 259-284.

Lima, B. A., Almeida, B. R. S., Sousa, E. A. B., Silva Cruz, G., Melo, M. B., Oliveira Melo, L., \& Santos, M. F. (2018). Estrutura e dinâmica florestal sob efeito do manejo madeireiro na Flona Tapajós. Advances in Forestry Science, 5(4), 437-443.

Lima, R. B., Rutishauser, E., Silva, J. A. A., Guedes, M. C., Herault, B., Oliveira, C. P., \& Ferreira, R. L. C. (2021). Accurate estimation of commercial volume in tropical forests. Forest Science, 67(1), 14-21.

Magurran, A. E. (1988). Ecological diversity and its measurement (179 p.). New Jersey: Princeton University Press. 
Mueller-Dombois, D., \& Ellenberg, H. (1974). Aims and methods of vegetation ecology (547 p.). New York: John Wiley \& Sons.

Oliveira Junior, R. C., Keller, M. M., Ramos, J. F. F., Beldini, T. P., Crill, P. M., Camargo, P. B., \& Van Haren, J. (2015). Chemical analysis of rainfall and throughfall in the Tapajos National Forest, Belterra, Pará, Brazil. An Interdisciplinary Journal of Applied Science, 10(2), 263-285.

Oliveira, E. K. B., Rezende, A. V., Freitas, L. J. M., Murta Júnior, L. S., Barros, Q. S., \& Costa, L. S. (2019). Monitoramento da estrutura e caracterização ecológica em floresta tropical manejada na Amazônia Brasileira. Revista Brasileira de Ciências Agrárias, 14(4), 1-12. http://dx.doi.org/10.5039/agraria.v14i4a6867.

Oliveira, L. C., Do Couto, H. T. Z., Silva, J. N. M., \& Carvalho, J. O. P. (2005). Efeito da exploração de madeira e tratamentos silviculturais na composição florística e diversidade de espécies em uma área de 136 ha na Floresta Nacional do Tapajós, Belterra, Pará. Scientia Forestalis, 69, 62-76.

Pereira, D., Santos, D., Vedoveto, M., Guimaraes, J., \& Verissimo, A. 2010. Fatos florestais da Amazônia (1. ed., 124 p.). Belém: Imazon.

Phillips, O. L., Hall, P., Gentry, A. H., Sawyer, S. A., \& Vasquez, R. (1994). Dynamics and species richness of tropical rain forests. Proceedings of the National Academy of Sciences of the United States of America, 91(7), 2805-2809.

Pielou, E. C. (1975) Ecological diversity (165 p.). New York: John Wiley \& Sons.

R Development Core Team. (2019). R: a language and environment for statistical computing. Vienna: $\mathrm{R}$ Foundation for Statistical Computing.

Razali, N. M., \& Wah, Y. B. (2011). Power comparisons of shapiro-wilk, kolmogorov-smirnov, lilliefors and anderson-darling tests. Journal of Statistical Modeling and Analytics, 2(1), 21-33.

Reis, L. P., Silva, J. N. M., Reis, P. C. M., Carvalho, J. O. P., Queiroz, W. T., \& Ruschel, A. R. (2013). Efeito da exploração de impacto reduzido em algumas espécies de Sapotaceae no leste da Amazônia. Floresta, 43(3), 395-406.

Ribeiro, R. B. D. S., Gama, J. R. V., Martins, S. V., Moraes, A., Santos, C. A. A. D., \& Carvalho, A. N. D. (2013), Estrutura florestal em projeto de assentamento, comunidade São Mateus, município de Placas, Pará, Brasil. Revista Ceres, 60(5), 610-620.

Ruschel, A. R. (2008). Dinâmica da composição florística e do crescimento de uma floresta explorada há 18 anos na flona Tapajós, PA (Documentos). Brasília: Embrapa Amazônia Oriental.

Rutishauser, E., Hérault, B., Petronelli, P., \& Sist, P. (2016). Tree height reduction after selective logging in a tropical forest. Biotropica, 48(3), 285-289.

Santos, M. F. D., Gama, J. R. V., Figueiredo Filho, A., Costa, D. L. D., Retslaff, F. A. D. S., Silva-Ribeiro, R. B. D., \& Rode, R. (2019). Conicity and yield for lumber from commercial species from the Amazon. Cerne, 25(4), 439-450.

Schwartz, G., Peña-Claros, M., Lopes, J. C. A., Mohren, G. M. J., \& Kanashiro, M. (2012). Midterm effects of reduced-impact logging on the regeneration of seven tree comercial species in the Eastern Amazon. Forest Ecology and Management, 274, 116-125.

Silva, E. J. V. (2004). Dinâmicas de florestas manejadas e sob exploração convencional na Amazônia oriental (Tese de doutorado). Escola de Engenharia de São Carlos, Universidade de São Paulo, São Carlos.

Silva, J. N. M., \& Araújo, S. M. (1984). Equação de volume para árvores de menor diâmetro na floresta nacional do tapajós. Boletim de Pesquisa Florestal., 8-9, 16-25.

Silva, J. N. M., Carvalho, J. O. P., Lopes, J. C. A., \& Carvalho, M. S. P. (1984). Equação de volume para a Floresta Nacional do Tapajós. Boletim de Pesquisa Florestal., 8/9, 50-63.

Silva, J. N. M., Carvalho, J. O. P., Lopes, J., De Almeida, B. F., Costa, D. H. M., Oliveira, L. C., Vanclay, J. K., \& Skovsgaard, J. P. (1995). Growth and yield of a tropical rain forest of the brazilian Amazon 13 years after logging. Forest Ecology and Management, 71(3), 267-274.

Silva, J. N. M., Lopes, J. C. A., Oliveira, L. C., Silva, S. M. A., Carvalho, J. O. P., Costa, D. H. M., Melo, M. S., \& Tavares, M. J. M. (2005). Diretrizes para instalação e medição de parcelas permanentes em florestas naturais da Amazônia Brasileira. Belém: Embrapa Amazônia Oriental.

Sist, P., \& Ferreira, F. N. (2007). Sustainability of reduced-impact logging in the Eastern Amazon. Forest Ecology and Management, 243(2-3), 199-209.

Souza, D. R., Souza, A. L., Leite, H. G., \& Yared, J. A. G. (2006). Análise estrutural em floresta ombrófila densa de terra firme não explorada, Amazônia Oriental. Revista Árvore, 30(1), 75-87. 
Ter Steege, H., Pitman, N. C., Sabatier, D., Baraloto, C., Salomão, R. P., Guevara, J. E., Phillips, O. L., Castilho, C. V., Magnusson, W. E., Molino, J.-F., Monteagudo, A., Nunez Vargas, P., Montero, J. C., Feldpausch, T. R., Coronado, E. N. H., Killeen, T. J., Mostacedo, B., Vasquez, R., Assis, R. L., Terborgh, J., Wittmann, F., Andrade, A., Laurance, W. F., Laurance, S. G. W., Marimon, B. S., Marimon, B.-H., Guimaraes Vieira, I. C., Amaral, I. L., Brienen, R., Castellanos, H., Cardenas Lopez, D., Duivenvoorden, J. F., Mogollon, H. F., Matos, F. D. A., Davila, N., Garcia-Villacorta, R., Stevenson Diaz, P. R., Costa, F., Emilio, T., Levis, C., Schietti, J., Souza, P., Alonso, A., Dallmeier, F., Montoya, A. J. D., Fernandez Piedade, M. T., Araujo-Murakami, A., Arroyo, L., Gribel, R., Fine, P. V. A., Peres, C. A., Toledo, M., Aymard C, G. A., Baker, T. R., Ceron, C., Engel, J., Henkel, T. W., Maas, P., Petronelli, P., Stropp, J., Zartman, C. E., Daly, D., Neill, D., Silveira, M., Paredes, M. R., Chave, J., Lima Filho, D. A., Jorgensen, P. M., Fuentes, A., Schongart, J., Cornejo Valverde, F., Di Fiore, A., Jimenez, E. M., Penuela Mora, M. C., Phillips, J. F., Rivas, G., van Andel, T. R., von Hildebrand, P., Hoffman, B., Zent, E. L., Malhi, Y., Prieto, A., Rudas, A., Ruschell, A. R., Silva, N., Vos, V., Zent, S., Oliveira, A. A., Schutz, A. C., Gonzales, T., Trindade Nascimento, M., Ramirez-Angulo, H., Sierra, R., Tirado, M., Umana Medina, M. N., van der Heijden, G., Vela, C. I. A., Vilanova Torre, E., Vriesendorp, C., Wang, O., Young, K. R., Baider, C., Balslev, H., Ferreira, C., Mesones, I., Torres-Lezama, A., Urrego Giraldo, L. E., Zagt, R., Alexiades, M. N., Hernandez, L., Huamantupa-Chuquimaco, I., Milliken, W., Palacios Cuenca, W., Pauletto, D., Valderrama Sandoval, E., Valenzuela Gamarra, L., Dexter, K. G., Feeley, K., LopezGonzalez, G., \& Silman, M. R. (2013). hyperdominance in the amazonian tree flora. Science, 342(6156), 1243092.

Vatraz, S., Carvalho, J. O. P., Silva, J. N. M., \& Castro, T. C. (2016). Efeito da exploração de impacto reduzido na dinâmica do crescimento de uma floresta natural. Scientia Forestalis, Piracicaba, 44(109), 261-271.

Vidal, E., Viana, V. M., \& Batista, J. L. F. (2002). Crescimento de floresta tropical três anos após colheita de madeira com e sem manejo florestal na Amazônia oriental. Scientia Forestalis, 61(1), 133-143.

Vieira, D. S., Gama, J. R. V., Ribeiro, R. B. S., Ximenes, L. C., Corrêa, V. V., \& Alves, A. F. (2014). Comparação estrutural entre floresta manejada e não manejada na Comunidade Santo Antônio, Estado do Pará. Ciência Florestal, 24(4), 1067-1074.

Xaud, H. A. M., Martins, F. S. R. V., \& Santos, J. R. (2013). Tropical forest degradation by mega-fires in the northern Brazilian Amazon. Forest Ecology and Management, 294, 97-106.

Contribuição dos autores: BAL: Curadoria de Dados, Metodologia, Investigação, Escrita - Primeira Redação, Escrita - Revisão e Edição; MFN: Análise Formal, Metodologia, Supervisão; TFS e GSC: Supervisão, Visualização; LOM: Curadoria de Dados. 